\title{
Transversality Condition in Continuum Mechanics
}

\author{
Jianlin Liu \\ Department of Engineering Mechanics, China University of Petroleum, \\ China
}

\section{Introduction}

Nature creates all kinds of miraculous similar phenomena in the real world. For example, the spiral morphologies exist in nebula, sunflower seed array, grapevine, and DNA. There are also a lot of similarities in physical theories and principles, such as the analogy between a harmonic vibration system and an RLC oscillation circuit, between a membrane and a sand-heap in elasticity and plasticity, and between fluid mechanics and electricity or magnetism. The great scientist Maxwell pointed out that the form of the capillary surface is identical with that of the elastic curve, which was later tested by the experiment of Clanet and Quere (2002), and then was analyzed by Liu in detail (2009). Exploring these similarities and analogies can help us understand the underlining secret of nature, and pave the way to incorporate several similar phenomena into a unified analysis frame.

For this study, we mainly focus on the similarity in the adhesion of materials and devices at micro and nano scales, which may be caused by van der Waals force, Casimir force, capillary force or other interaction forces. Among others, the adhesion of a slender structure as micro-beam or carbon nanotube (CNT) is of great value for both theoretical and practical aspects. In these systems, due to considerable surface to volume ratio in low-dimensional micro/nano-systems, surface tension or interfacial energy will dominate over the volume force as their dimensions shrink to micro/nano-meters, which presents a lot of novel behaviors distinct with those of the macroscopic systems (Poncharal, et al., 1999). The typical phenomenon is stiction of the micro-beams, such as the micro/nano-wires and micro/nano-belts which are widely used as building blocks of micro-sensors, resonators, probes, transistors and actuators in M/NMES (micro/nano-electro-mechanical systems). In micro-contact printing technology, adhesion associated with van der Waals force leads to stamp deformation (Hui, et al., 2002), and the micro-machined MEMS structures will spontaneously adhere on the substrate under the influence of solid surface energy or liquid surface tension (Zhao, et al., 2003). This failure due to stiction has become a major limitation to push the better application of these novel engineering devices, and the problem has been highlighted as a hot topic in the past decades. The main reason of stiction is that in the small spacings, the slender structures with high compliance are easily brought into contact with the substrate of strong surface energy.

Another related issue is the self-collapse of a single wall carbon nanotube (SWCNT), in which process its initially circular cross-section will jump to a flat ribbon like shape. The 
reason lies in that CNTs capture the characteristic of hollow cylindrical structures, which render them susceptible to lateral deformation. In reality, this morphology was first observed and explored by transmission electron microscopy (TEM) (Ruoff, et al., 1993; Chopra, et al., 1995) and then by AFM (Martel, et al., 1998; Yu, et al., 2000). To date, much effort has been devoted towards understanding the mechanism of CNT collapse. Gao et al. (1998) used molecular dynamic (MD) simulations to discover that there are two possible configurations for a CNT in equilibrium state, i.e. the circular one and the collapsed one. For nanotubes with radius in the range of $R<R_{\min }$ only the circular configuration exits. When the radius satisfies $R_{\min }<R<R_{\max }$, both of the shapes exist, and the collapsed tube is in a metastable state. For the radius $R>R_{\max }$, the collapsed configuration is energetically favorable and thermodynamically stable. Their results also exhibited that the critical radii are insensitive to the chirality of the tube, and the values of the critical radii are $R_{\min } \approx 1.1$ $\mathrm{nm}$ and $R_{\max } \approx 3 \mathrm{~nm}$. Subsequently, Pantano et al. (2004) adopted a continuum approach and finite element method to investigate the morphology of the collapsed CNTs. In succession, Tang et al. studied the collapse of nanotubes using an inextensible elastica model. In use of phase plane analysis, they showed that CNTs can take collapsed configurations of different orders (Tang and Glassmaker, 2010). Recently, they investigated the energetics of selfcollapse of a single CNT by using the continuum mechanics method, and calculated the critical radii $R_{\min } \approx 0.699 \mathrm{~nm}$ and $R_{\max } \approx 0.976 \mathrm{~nm}$ (Tang, et al., 2005b). This significant difference from the result of Gao et al. is because a distinct force field and physical parameters were selected, which greatly affect the results of analytical solutions and molecular simulations. However, Liu et al. (2004) performed simulations on the formation of fully collapsed SWCNTs with the atomic scale finite element method, and proposed that for armchair SWCNTs, collapse occurs for the critical radius (for $\mathrm{n}=30$ ) is $R_{\max } \approx 2.06 \mathrm{~nm}$, which is also different from the aforementioned results. In fact, this value of critical radius of 2.06 $\mathrm{nm}$ was verified by TEM observations, which demonstrated that there exists a collapsed SWCNT of $2.5 \mathrm{~nm}$ in radius (Xiao, et al., 2007). In spite of the above cited studies on the collapse of CNTs, there are hitherto no analytical solution for the collapse problem of CNTs, which involves large deformation and strong geometric nonlinearity.

Although belonging to different phenomena, we strongly stress that, the adhesion of microbeams and collapse of SWCNT can be actually incorporated into a unified analysis frame. Based upon this frame, we can easily calculate the parameters of adhesion for different systems. The outline of this article is organized as follows. In Section 2, we established the formulations of energy functional, and derived the governing equation and transversality condition in consideration of the moving boundary. In Section 3, in use of the constructed frame, we obtained the critical adhesion length of two micro-beams stuck by a thin liquid film, and the deflections of the beams. In Section 4, we calculated the critical radii and collapsed shapes of SWCNTs via the classical elastica solution, which was derived from the energy functional.

\section{Energy functional and transversality condition}

We first provide the analysis of the scaling law of a system with different energy originations. The typical length of the slender structure is denoted as $L_{c}$, then the interfacial or surface energy $U_{S} \propto L_{c}$, the elastic strain energy $U_{E} \propto L_{c}^{2}$, and the potential energy due to gravity $U_{G} \propto L_{c}^{3}$ (Roman and Bico, 2010). As the dimension of a macroscopic structure 
shrinks to nanometers, the effect of surface energy will become significant and should be taken into account. In this study, we assume that the gravity effect is negligible, for the interplay between the surface energy and elasticity is predominant.

Let us consider a generalized elastic system denoted by a continuous and smooth curve, where part of the curve is adhered by some special interfacial forces. The position of an arbitrary point in the curve is schematized by the arc length $s$, the total length of the curve is $L$, and the segment length dealing with the elastic deformation is $a$. The kernel problem is how to determine the unknown length $a$ in the equilibrium state according to the principle of least potential energy.

The functional of the total potential energy about the system is normally written as:

$$
\Pi[y(x, a)]=\int_{0}^{a} U_{E} \mathrm{~d} x-\int_{a}^{L} W_{a} \mathrm{~d} x,
$$

where the first term in the right side of Eq. (1) is strain energy, $U_{E}=F\left[x, y(x, a), y^{\prime}(x, a), y^{\prime \prime}(x, a)\right]$, and the symbols ()$^{\prime}=\frac{\partial()}{\partial x},()^{\prime \prime}=\frac{\partial^{2}()}{\partial x^{2}}$.

The second term in the right side of Eq. (1) is named as the work of adhesion between two surfaces, which is normally expressed as (Tang, et al., 2005a)

$$
W_{a}=\left(\gamma_{1}+\gamma_{2}-\gamma_{12}\right) b
$$

where $b$ is the contact width out of the curve, $\gamma_{1}$ and $\gamma_{2}$ are the surface energies of the two different phases, and $\gamma_{12}$ the interfacial energy. In the conventional definition, the work of adhesion is actually the work per unit area necessary to create two new surfaces from a unit area of an adhered interface, which is a positive constant for any two homogeneous materials binding at an interface at a fixed temperature. For the two phases are of the same material, the work of adhesion degenerates to the cohesive work

$$
W_{c}=2 \gamma_{1} b
$$

At micro and nano scales, the cohesive work is normally termed as the binding energy $E_{B}$. Furthermore, for the interface consisting of a thin liquid film, the expression of the cohesive work is (Bico, et al., 2004)

$$
W_{c}=2\left(\gamma_{\mathrm{SV}}-\gamma_{\mathrm{SL}}\right) b=2 \gamma \cos \theta_{\mathrm{Y}} b,
$$

where $\gamma_{\mathrm{SV}}, \gamma_{\mathrm{SL}}, \gamma$ are the interfacial tensions of solid/vapor, solid/liquid and liquid/vapor interfaces, respectively, with $\theta_{Y}$ being the Young's contact angle of the liquid. In the above derivation, the Young's equation is employed.

We should mention that, the energy functional of Eq. (1) actually includes two variables, namely, the function $y$ and $a$, because $a$ is yet an unknown when solving the governing equation. This results to an intractable problem, for the undetermined variable $a$ causes the bound movement of the system, which should be considered as a movable boundary condition problem during variation process. Therefore, in use of the principle of least potential energy, one obtains the following variational result 


$$
\delta \Pi[y(x, a)]=\delta \Pi_{1}+\delta \Pi_{2}=0,
$$

where

$$
\begin{gathered}
\delta \Pi_{1}=\int_{0}^{a}\left(F_{y} \delta y+F_{y^{\prime}} \delta y^{\prime}+F_{y^{\prime \prime}} \delta y^{\prime \prime}\right) \mathrm{d} x \\
=\left[F_{y^{\prime}} \delta y+F_{y^{\prime \prime}} \delta y^{\prime}-\frac{\partial}{\partial x} F_{y^{\prime \prime}} \delta y\right]_{0}^{a}+\int_{0}^{a}\left(F_{y}-\frac{\partial}{\partial x} F_{y^{\prime}}+\frac{\partial^{2}}{\partial x^{2}} F_{y^{\prime \prime}}\right) \delta y \mathrm{~d} x .
\end{gathered}
$$

For the other portion of variation, in consideration of the moving boundary at $s=a$, we then focus on the transversality condition in mathematical meaning, and show how to derive its expression. We first revisit the derivative definition about an integration including a parameter $\alpha$. Let

$$
\phi(\alpha)=\int_{a(\alpha)}^{b(\alpha)} F(x, \alpha) \mathrm{d} x
$$

and then we have its derivative

$$
\phi^{\prime}(\alpha)=\int_{a(\alpha)}^{b(\alpha)} F_{\alpha}(x, \alpha) \mathrm{d} x+F[b(\alpha), \alpha] b^{\prime}(\alpha)-F[a(\alpha), \alpha] a^{\prime}(\alpha) .
$$

Similarly, considering the moving boundary at $s=a$, we can obtain the second part of variation on the functional in Eq. (1), that is

$$
\delta \Pi_{2}=\left[F-y^{\prime} F_{y^{\prime}}-y^{\prime \prime} F_{y^{\prime \prime}}+y^{\prime} \frac{\mathrm{d} F_{y^{\prime \prime}}}{\mathrm{d} x}+W\right]_{x=a} \delta a .
$$

In the above derivations, the partial derivative of the function $F$ is designated as $F_{()}=\frac{\partial F}{\partial()}$. The forced or fixed boundary conditions are normally prescribed as

$$
y(0)=y_{0}, y^{\prime}(0)=y_{0}^{\prime} ; y(a)=y_{a}, y^{\prime}(a)=y_{a}^{\prime} .
$$

Inserting Eq. (6) and (9) into (5), and according to the arbitrariness of the variation, one can get the governing differential equation, i.e. the Euler-Poisson equation:

$$
F_{y}-\frac{\partial}{\partial x} F_{y^{\prime}}+\frac{\partial^{2}}{\partial x^{2}} F_{y^{\prime \prime}}=0
$$

Besides the above equation, the arbitrariness of variation about $a$ leads to

$$
W_{a}=\left[y^{\prime} F_{y^{\prime}}+y^{\prime \prime} F_{y^{\prime \prime}}-y^{\prime} \frac{\mathrm{d} F_{y^{\prime \prime}}}{\mathrm{d} x}-F\right]_{x=a} .
$$

In fact, the concept of energy release rate or $J$ integral was also adopted to investigate the problem of moving boundary (Tang et al., 2005a, 2005b). However, the exact solution of the energy release rate is often impossible to acquire, as a result, one can avoid this way and 
apply the transversality condition to determine the unknown $a$. This thought paves a new way to solve this kind of problems dealing with movable boundary. In addition, the transversality condition is essential to calculate the contact angle of a droplet, the morphology of a cell, and the peeling of a CNT from the substrate (Oyharcalbal and Frisch, 2005; Bormasshenko and Whyman, 2008; Seifert, 1990). We will then put to use this method to analyze some practical topics, and the adhesion of two micro-beams and the collapsed shape of a single wall carbon nanotube (SWCNT) are selected as study cases.

\section{Adhesion of two micro-beams}

Let us consider two identical micro-beams with the same Young's modulus $E$, and moment of inertia on the cross section $I$, which are stuck together by the interfacial energy $W_{a}$ (or work of adhesion) due to a thin liquid film. The cross section of the beam is a rectangle, with the width $b$, and thickness $e$, then $I=b e^{3} / 12$. Refer to a Cartesian coordinate system $(o-x y)$. As shown in Fig. 1, the distance between the ends of the two beams is $d$, the detached segment length is $L_{\mathrm{dry}}$, the adhered part is $L_{\mathrm{wet}}$, and the total length of the beam is $L$.
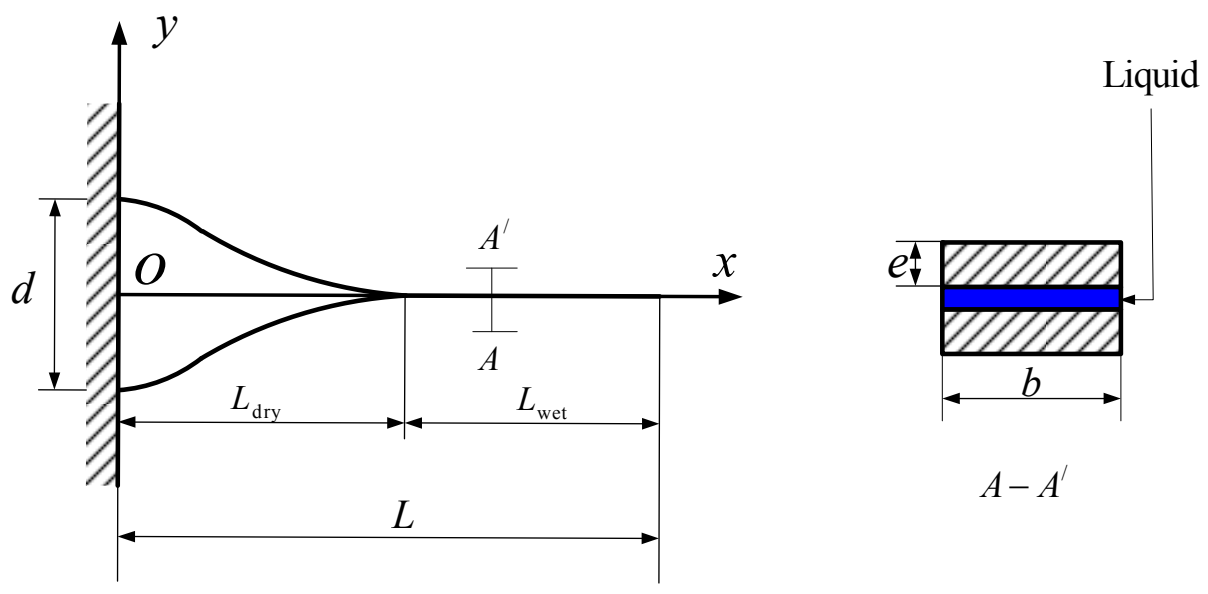

Fig. 1. Capillary adhesion of two beams with rectangular cross-sections.

According to Eqs. (1) and (4), the total potential energy of the two beam system can be easily expressed as (Bico, et al., 2004; Liu and Feng, 2007a)

$$
\Pi=E I \int_{0}^{L_{\mathrm{dry}}} w^{\prime \prime 2} \mathrm{~d} x-2 \gamma \cos \theta_{Y}\left(L-L_{\mathrm{dry}}\right) .
$$

In use of Eqs. (11) and (12), one can deduce the governing equation

$$
w^{(4)}=0,
$$

and the transversality condition at the moving bound

$$
2 \gamma \cos \theta_{\mathrm{Y}} b=\operatorname{EIw}\left(L_{\mathrm{dry}}\right)^{2} .
$$

The fixed boundary conditions of a single beam are specified as 


$$
w(0)=d / 2, w^{\prime}(0)=0 ; w\left(L_{\mathrm{dry}}\right)=0, w^{\prime}\left(L_{\mathrm{dry}}\right)=0,
$$

Then the corresponding solution of Eq. (14) is written as

$$
w=\frac{d}{L_{\mathrm{dry}}^{3}} x^{3}-\frac{3 d}{2 L_{\mathrm{dry}}^{2}} x^{2}+\frac{d}{2} .
$$

The combination of Eq. (15) and (17) yields

$$
L_{\mathrm{dry}}=\sqrt[4]{\frac{3 E e^{3} d^{2}}{8 \gamma \cos \theta_{Y}}} .
$$

If the total length of the beam $L<L_{\mathrm{dry}}$ or $\tilde{L}_{\mathrm{dry}}=L_{\mathrm{dry}} / L>1$, the adhesion energy induced by the introduction of a liquid film between the two beams is insufficient to provide the strain energy of deformation, and therefore, the two beams will not adhere. On the contrary, if $\tilde{L}_{\text {dry }}<1$, the surface energy is larger than the strain energy, and then the adhesion of the two beams is possible.

Equation (18) also requires that the contact angle $\theta_{Y}$ must satisfy $0 \leq \theta_{Y}<\pi / 2$, that is, the beams must be hydrophilic. In other words, capillary adhesion cannot happen between two hydrophobic hairs. The critical length $L_{\text {dry }}$ for capillary adhesion of two beams increases with the decreasing of the surface tension and the Young's contact angle $\theta_{Y}$ in the range of $0 \leq \theta_{Y}<\pi / 2$, and with the increasing of the end distance $d$ of the two beams. In addition, the deflection diagrams of the two beams can be determined easily from Eq. (17) and are plotted in Fig. 2 for several representative values of the interbeam spacing, $d=0.5,1.0$ and $2.0 \mathrm{~mm}$, where we take the following parameters: $E I / b=5.1 \times 10^{-4} \mathrm{~N} \cdot \mathrm{m}, \theta_{\mathrm{Y}}=10^{\circ}$ and $\gamma=72 \times 10^{-3} \mathrm{~N} / \mathrm{m}$.

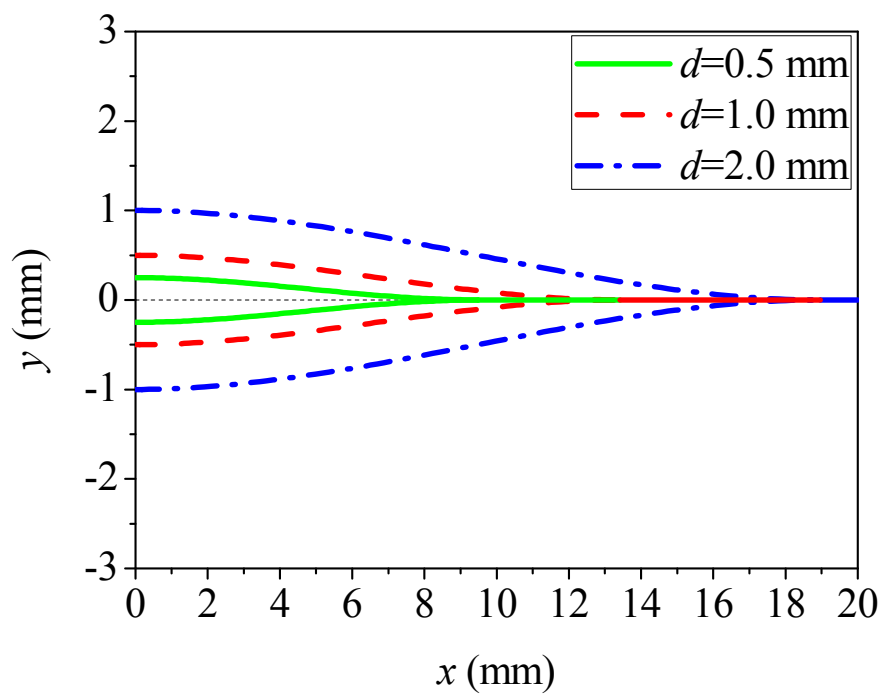

Fig. 2. Deflections of two adhered micro-beams by the liquid film. 


\section{Collapsed morphology of a SWCNT}

We then put to use our analysis frame to examine the collapsed morphology of a SWCNT, which is initially circular with a radius $R$ and an axial length $L$. The current configuration incorporates a flat contact zone in the middle part and two non-contact regions at the ends, as shown in Fig. 3. In essence, this configuration is stabilized by the van der Waals interplay between the upper and lower portion of the CNT walls, primarily within the horizontal contact zone, because the van der Waals force decays rapidly in the non-contact areas. As a reasonable simplification, the van der Waals force between the upper and lower portion of the CNT walls in the non-contact domain is ignored in our calculation. Normally, the van der Waals force between two carbon atoms is repulsive at a very close range, so the CNT wall contact is defined by an equilibrium separation $d_{0}$ between the flat regions. The distance between the flat contact zone and the extreme point of the CNT is denoted as $b$. From the experimental picture, we can see that the collapsed shape of CNT is symmetric, which was also verified by the molecular simulations (Tang, et al. 2005b).
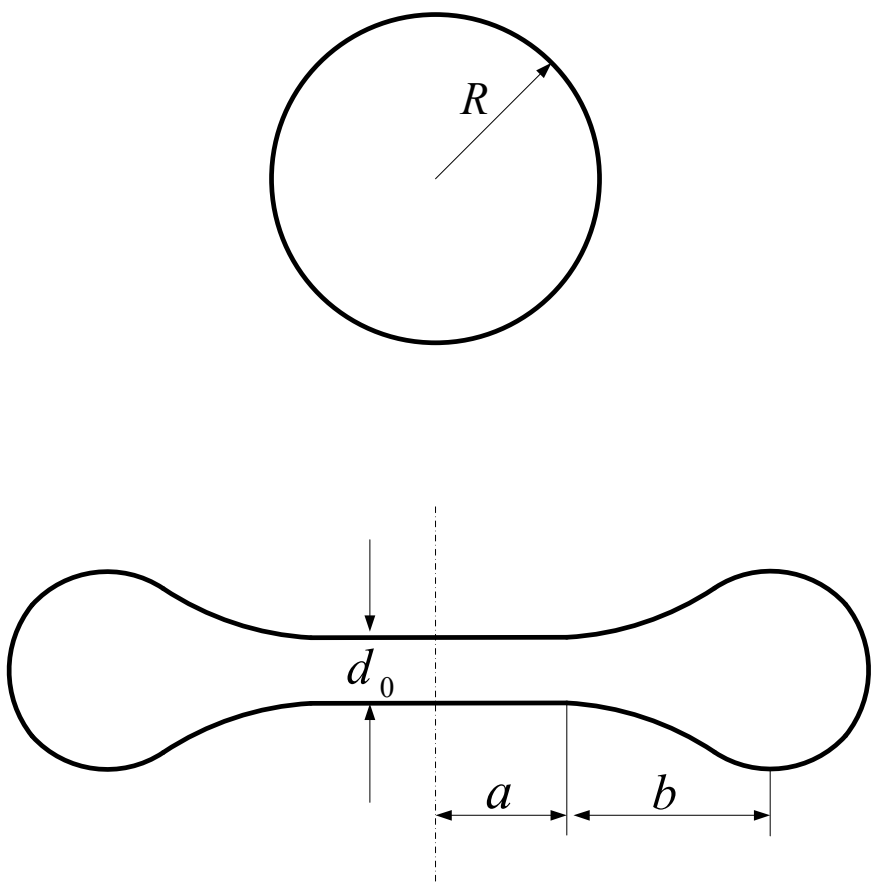

Fig. 3. Carbon nanotubes with a circular shape of the radius $R$, and with a collapsed shape. The semi-width of the flat contact zone of the collapsed CNT is $a$, and the separation distance is $d_{0}$.

Due to the symmetry and smoothness of this configuration, a quarter of the structure is selected and then modeled as a plate or an elastica with two clamped ends, as schematized in Fig. 4. This famous elastica theory, which can be traced back to the historic contribution of Euler (Love, 1906), has been successfully used to solve some finite deformation problems of slender structures (Bishopp, 1945; Liu and Feng, 2007a; Glassmaker and Hui, 2004). 


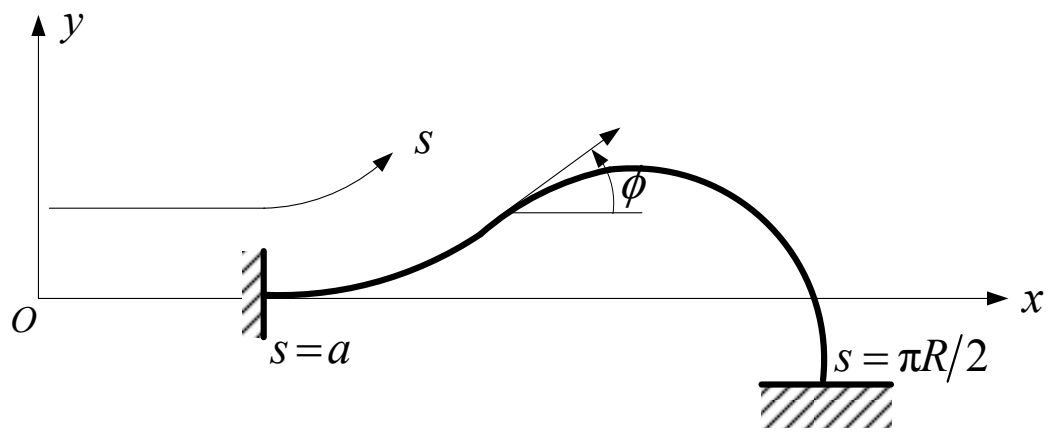

Fig. 4. Elastica model for a quarter of the CNT, with the arc length $s$ and slope angle $\phi$ ranging from 0 to $-90^{\circ}$.

In fact, there is another underling assumption that, the deformation along the axis of the nanotube is uniform, which has already been verified by experiments and MD simulations (Ruoff, et al. 1993; Chopra, et al., 1995; Pantano, 2004; Tang, et al., 2005b). As a result, we select the cross section representing the whole tube, and model the thin wall as a curvilinear abscissa. The total in-plane length of the elastica is thus $\pi R / 2$, and the adhered segment is $a$. Refer to a Cartesian coordinate system (o-xy). Besides the Euler coordinate $x$ and $y$, the arc length $s$, being a Lagrange coordinate, is also employed as a variable in our analysis. The slope angle of the elastica at an arbitrary point is $\phi$, which continuously changes from $0^{\circ}$ at its left end to $-90^{\circ}$ at the right end. The bending stiffness of the elastica is $E I$, where $I$ is the inertial moment of the cross section, and $E=\bar{E} /\left(1-v^{2}\right)$, with $\bar{E}$ being the Young's modulus and $v$ the Poisson's ratio of the material.

According to the elastica model of Fig. 4, the fixed displacement boundary conditions of the system are specified as

$$
\phi(a)=0, \phi\left(\frac{\pi R}{2}\right)=-\frac{\pi}{2}, \phi(\pi R-a)=-\pi ; y(a)=0, y\left(\frac{\pi R}{2}\right)=-\frac{d_{0}}{2} .
$$

The additional geometric conditions of the elastica are

$$
\dot{x}=\cos \phi, \dot{y}=\sin \phi,
$$

where the dot above a parameter stands for its derivative with respect to the arc length $s$. In consideration of the symmetry of this configuration, the total potential energy functional of the system can be written as

$$
\Pi=2 L \int_{a}^{\pi R / 2}\left[E I \dot{\phi}^{2}+\lambda_{1}(\dot{x}-\cos \phi)+\lambda_{2}(\dot{y}-\sin \phi)\right] d s-2 W_{c} a L,
$$

where $\lambda_{1}$ and $\lambda_{2}$ are two Lagrange multipliers, enforcing the additionally geometrical relations of Eq. (20).

To deduce the expression of the cohesive work, we choose the van der Waals potential as (Tang, et al., 2005a) 


$$
V(r)=\varepsilon\left[3\left(\frac{r^{*}}{r}\right)^{6}-2\left(\frac{r^{*}}{r}\right)^{9}\right],
$$

where $\varepsilon=0.064 \mathrm{kcal} / \mathrm{mol}, r^{*}=0.401 \mathrm{~nm}$, and $r$ is the distance between two atoms. In this case, the cohesive work is the work per unit area to separate the two parallel graphite sheets from $d$ to $\infty$, which can be calculated via integration of Eq. (22):

$$
W(d)=\pi \rho^{2} \varepsilon\left[\frac{3\left(r^{*}\right)^{6}}{2 d^{4}}-\frac{4\left(r^{*}\right)^{9}}{7 d^{7}}\right],
$$

where $\rho$ is the number of carbon atoms per unit area, with the value of $\rho \approx 0.004 \mathrm{~nm}^{-2}$. In the experiment (Ruoff, et al. 1993), the equilibrium distance is measured as $d_{0}=0.338 \mathrm{~nm}$, and the corresponding cohesive work is calculated as $W_{c}=0.388627 \mathrm{~J} / \mathrm{m}^{2}$ according to Eq. (23).

Taking variation of the potential energy functional of Eq. (21), and in use of the principle of least potential energy, one has

$$
\delta \Pi=\delta \Pi_{1}+\delta \Pi_{2}=0
$$

where

$$
\begin{gathered}
\delta \Pi_{1}=2 L \int_{a}^{\pi R / 2}\left[2 E I \dot{\phi} \delta \dot{\phi}+\delta \lambda_{1}(\dot{x}-\cos \phi)+\delta \lambda_{2}(\dot{y}-\sin \phi)\right. \\
\left.+\lambda_{1} \delta \dot{x}+\lambda_{1} \sin \phi \delta \phi+\lambda_{2} \delta \dot{y}-\lambda_{2} \cos \phi \delta \phi\right] \mathrm{d} s \\
=2 L \int_{a}^{\pi R / 2}\left[-2 E I \ddot{\phi} \delta \phi+\delta \lambda_{1}(\dot{x}-\cos \phi)+\delta \lambda_{2}(\dot{y}-\sin \phi)\right. \\
\left.+\lambda_{1} \sin \phi \delta \phi-\lambda_{2} \cos \phi \delta \phi\right] d s+2 L\left[2 E I \dot{\phi} \delta \phi+\lambda_{1} \delta x+\lambda_{2} \delta y\right]_{a}^{\pi R / 2} \\
=2 L \int_{a}^{\pi R / 2}\left[\delta \lambda_{1}(\dot{x}-\cos \phi)+\delta \lambda_{2}(\dot{y}-\sin \phi)\right. \\
\left.-\left(2 E I \ddot{\phi}-\lambda_{1} \sin \phi+\lambda_{2} \cos \phi\right) \delta \phi\right] \mathrm{d} s-2 L \lambda_{1} \delta a
\end{gathered}
$$

In the above derivations, Eqs. (19) and (20) have been adopted.

Considering the moving boundary, the second part of the variation is expressed as

$$
\begin{gathered}
\delta \prod_{2}=-2 L\left[E I \dot{\phi}^{2}+\lambda_{1}(\dot{x}-\cos \phi)+\lambda_{2}(\dot{y}-\sin \phi)-2 \dot{\phi}(E I \dot{\phi})-\dot{x} \lambda_{1}-\dot{y} \lambda_{2}\right]_{s=a} \delta a \\
-2 W_{c} L \delta a \\
=2 L\left[E I \dot{\phi}(a)^{2}-W_{c}+\lambda_{1}\right] \delta a .
\end{gathered}
$$


Noticing the arbitrariness of the variation, one obtains the following governing equation

$$
2 E I \ddot{\phi}-\lambda_{1} \sin \phi+\lambda_{2} \cos \phi=0,
$$

where the Lagrange multipliers $\lambda_{1}$ and $\lambda_{2}$ can be easily identified as the horizontal and vertical internal forces in the elastica. The governing equation in Eq. (27) conforms to that derived by Tang et al. (Tang and Glassmaker, 2010), who adopted the method of force equilibrium.

Combination of Eqs. (24) and (26) leads to the additional boundary condition at the moving point

$$
E I \dot{\phi}(a)^{2}-W_{c}=0
$$

which is termed as the transversality condition. This additional condition represents the balance of the elastic strain energy and the van der Waals potential energy. It is worthy of being mentioned that the movable boundary condition in Eq. (28) can also be deduced via the concept of $J$ integral in fracture mechanics, as described by Glassmaker and Hui (2004) in their analysis of silicon-germanium nanotube formation.

Multiplying $\dot{\phi}$ to both sides of Eq. (27) and by integration, one has

$$
E I \dot{\phi}^{2}=D-\lambda_{1} \cos \phi-\lambda_{2} \sin \phi,
$$

where $D$ is an integration constant. Making use of Eq. (19), one obtains

$$
E I \dot{\phi}(a)^{2}=D
$$

It is noticed that the symmetry of the configuration verifies the relation of $\dot{\phi}(a)^{2}=\dot{\phi}(\Pi R-a)^{2}=D$.

Inserting Eq. (28) into (30), one has

$$
D=W_{c} .
$$

Introducing the parameter $C$ and $\alpha=\sqrt{\lambda_{2} /(2 E I)}$, we can obtain $\alpha^{2} C=D /(2 E I)$. Thus the governing equation (29) and transversality condition (30) are respectively transformed into

$$
\begin{gathered}
\frac{1}{2} \dot{\phi}^{2}=\alpha^{2}(C-\sin \phi), \\
\frac{1}{2} \dot{\phi}(a)^{2}=\alpha^{2} C .
\end{gathered}
$$

The combination of Eqs. (30) and (33) yields

$$
\alpha=\frac{1}{L_{e c} \sqrt{2 C}} .
$$


Here, we have defined a new characteristic length, i.e. the elasto-cohesive length $L_{e c}$ $=\sqrt{E I / W_{c}}$, which is different from the elasto-capillary length $L_{E C}$ named by Bico et al. (2004) and Roman (2010). For a slender structure adhered by a liquid film, the elasto-cohesive length $L_{e c}=\frac{\sqrt{2}}{2} L_{E C}$ when $\theta_{Y}=0$. It is seen that in this case, Eqs. (30) and (34) are consistent with the results in the reference (Bico, et al., 2004).

Note that the arc increment $\mathrm{d} s$ is always positive and the increasing of the slope angle is not monotonic, and Eq. (32) is simplified as

$$
\alpha \mathrm{d} s=\frac{|\mathrm{d} \phi|}{\sqrt{2(C-\sin \phi)}} .
$$

For convenience of integration, the variable $\phi$ should be replaced with another variable $\theta$. These two variables are related by

$$
(1+C) \sin ^{2} \theta=2 k^{2} \sin ^{2} \theta=1+\sin \phi \quad(0 \leq \theta \leq \pi, k>0),
$$

and

$$
\begin{gathered}
\sqrt{2(C-\sin \phi)}=2 k|\cos \theta| \\
|\mathrm{d} \phi|=\frac{2 k|\cos \theta|}{\sqrt{1-k^{2} \sin ^{2} \theta}} \mathrm{d} \theta, \\
\frac{|\mathrm{d} \phi|}{\sqrt{2(C-\sin \phi)}}=\frac{\mathrm{d} \theta}{\sqrt{1-k^{2} \sin ^{2} \theta}} .
\end{gathered}
$$

Substituting Eqs. (34-39) into the prescribed displacement boundary condition in Eq. (19) leads to

$$
\begin{gathered}
\alpha y\left(\frac{\pi R}{2}\right)=\int_{0}^{-\frac{\pi}{2}} \frac{\sin \phi|d \phi|}{\sqrt{2(C-\sin \phi)}} \\
=F(k, \pi)-F\left(k, \theta_{0}\right)-2\left[E(k, \pi)-E\left(k, \theta_{0}\right)\right]=-\frac{d_{0}}{2 \sqrt{4 k^{2}-2} L_{e c}},
\end{gathered}
$$

where $\sin \theta_{0}=1 /(\sqrt{2} k), F(k, \theta)$ and $E(k, \theta)$ are the elliptic integrals of the first and second kinds, which are respectively defined as

$$
\begin{aligned}
& F(k, \theta)=\int_{0}^{\theta} \frac{1}{\sqrt{1-k^{2} \sin ^{2} \theta}} \mathrm{d} \theta, \\
& E(k, \theta)=\int_{0}^{\theta} \sqrt{1-k^{2} \sin ^{2} \theta} \mathrm{d} \theta .
\end{aligned}
$$


To close this mathematical formulation of the above problem, we then complement the inextensible condition of the elastic rod (Bishopp and Drucker, 1945), which is written as

$$
\begin{gathered}
\alpha\left(\frac{\pi R}{2}-a\right)=\alpha \int_{a}^{\pi R / 2} d s \\
=\int_{0}^{-\frac{\pi}{2}} \frac{|\mathrm{d} \phi|}{\sqrt{2(C-\sin \phi)}}=F(k, \pi)-F\left(k, \theta_{0}\right) .
\end{gathered}
$$

From Eq. (40), one can solve the corresponding values of $k=0.82$ for the given value of $d_{0}$. Then the substitution of Eq. (34) into (42) yields

$$
\frac{a}{\pi R}=\frac{1}{2}-\sqrt{4 k^{2}-2}\left[F(k, \pi)-F\left(k, \theta_{0}\right)\right] \frac{L_{e c}}{\pi R} .
$$

For a CNT with an initial radius $R=3 \mathrm{~nm}$, the analytical result of Eq. (43) is presented as 0.317854 , which is nearly equal to the approximated solution given by Tang et al. (2005b) is $a /(\Pi R) \approx\left(d_{0}-3.035 L_{e c}\right) /(\Pi R)+0.5=0.30485$.

After the flat contact length $a$ has been solved by Eq. (43), the deflection of the rod can be determined by

$$
\begin{gathered}
\alpha x=\alpha \int_{0}^{s} \cos \phi \mathrm{d} s=\alpha a+\sqrt{4 k^{2}-2}-2 k \cos \theta, \\
\alpha y=\alpha \int_{0}^{s} \sin \phi \mathrm{d} s=F(k, \theta)-F\left(k, \theta_{0}\right)-2\left[E(k, \theta)-E\left(k, \theta_{0}\right)\right] .
\end{gathered}
$$

The above displacements normalized by the elasto-cohesive length read

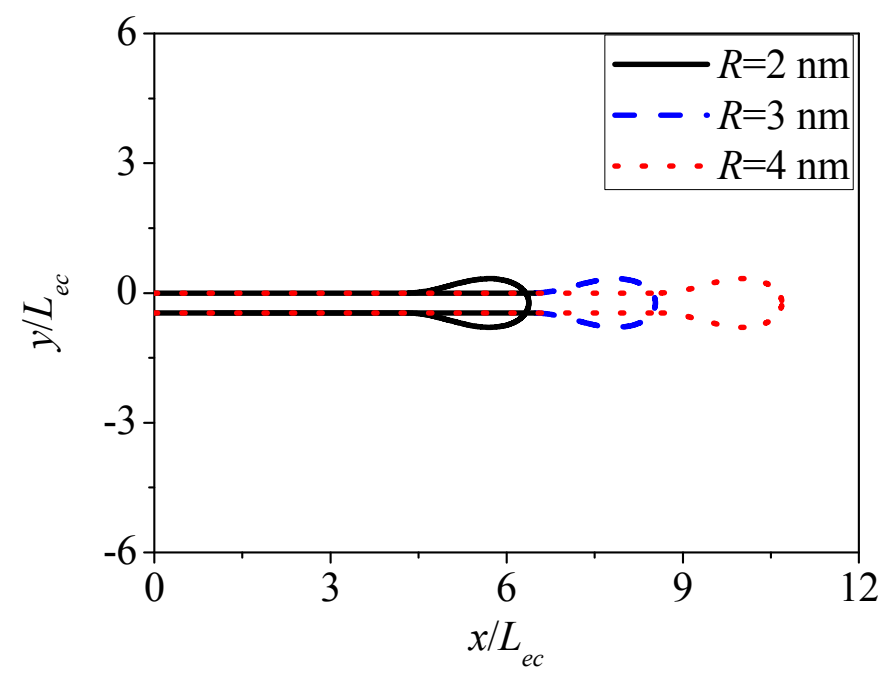

Fig. 5. Cross-section shapes of CNTs with initial radii of 2, 3 and $4 \mathrm{~nm}$, respectively. 


$$
\left\{\begin{array}{l}
\tilde{x}=\frac{\pi}{2} \tilde{R}-\sqrt{4 k^{2}-2}\left[F(k, \pi)-F\left(k, \theta_{0}\right)-\sqrt{4 k^{2}-2}+2 k \cos \theta\right], \\
\tilde{y}=\sqrt{4 k^{2}-2}\left\{F(k, \theta)-F\left(k, \theta_{0}\right)-2\left[E(k, \theta)-E\left(k, \theta_{0}\right)\right]\right\}
\end{array}\right.
$$

where $\tilde{x}=x / L_{e c}, \tilde{y}=y / L_{e c}$, and $\tilde{R}=R / L_{\mathrm{ec}}$.

Finally, the morphologies of three representative collapsed CNTs with $R=2,3$ and $4 \mathrm{~nm}$ are plotted in Fig. 5, where the parameters are the same as those in Fig. 3. It can be seen that, with increasing radius, the flat contact zone becomes larger, but the right ended shape does not change too much. Therefore, in the current calculation, the bending stiffness EI is selected as $2 \times 10^{-19} \mathrm{~N} \cdot \mathrm{m}$, and the corresponding elasto-cohesive length $L_{e c}=\sqrt{E I / W}=0.72 \mathrm{~nm}$.

\section{Conclusions}

In this study, we demonstrated that a lot of problems dealing with the moving boundaries can be grouped into a unified frame, such as the adhesion of micro-beams and collapse of SWCNT. We first constructed the energy functional of the general system, then derived the governing equation and the transversality condition. We put this analysis method to solve the critical length and deflections of two micro-beams. Moreover, we derived the governing equation, i.e., the elastica model of the collapsed morphology for the SWCNT. Under the inextensible condition of the rod, the closed-form solutions for the flat contact segment, critical radii, and collapsed configuration were obtained in terms of elliptical integrals. It is clearly shown that our analytical solutions are in good agreement with the results of the references.

This analysis method paves a new way to examine nano-scaled mechanics by means of continuum mechanics. The presented results are also beneficial to design and fabricate new devices, micro-sensors and advanced materials in micro/nano scale, which casts a light on enhancing their mechanical, chemical, optical and electronic properties. Furthermore, this model can be generalized to investigate both a macroscopic sheet wrapped by a liquid film and a CNT self-folded by van der Waals forces, and can be adopted to analyze the crack or contact problems.

\section{Acknowledgements}

The project was supported by the National Natural Science Foundation of China (10802099), Doctoral Fund of Ministry of Education of China (200804251520), and Natural Science Foundation of Shandong Province (ZR2009AQ006). The author also acknowledges the support from the Brain Korea 21 program at Seoul National University.

\section{References}

Bico, J., Roman, B., Moulin, L., Boudaoud, A. (2004) Adhesion: elastocapillary coalescence in wet hair. Nature, 432, pp. 9.

Bishopp, K. E., Drucker, D. C. (1945) Large deflections of cantilever beams. Quart. J. Appl. Math., 3, pp. 272-275.

Bormasshenko, E., Whyman, G. (2008) Variational approcach to wetting problems: calculation of a shape of sessile liquid drop deposited on a solid substrate in external field. Chem. Phys. Lett., 463, pp. 103-105. 
Chopra, N. G., Benedict, L. X., Crespi, V. H., Cohen, M. L., Louie, S. G., Zettl, A. (1995) Fully collapsed carbon nanotubes. Nature, 377, pp. 135-138.

Clanet, C., Quere, D. (2002) Onset of menisci. J. Fluid Mech., 460, 2002 460, pp. 131-149.

Gao, G., Cagin, T., Goddard III, W. A. (1998) Energetics, structure, mechanical and vibrational properties of single-walled carbon nanotubes. Nanotechnology, 9, pp. 184-191.

Glassmaker, N. J., Hui, C. Y. (2004) Elastica solution for a nanotube formed by self-adhesion of a folded thin film. J. Appl. Phys. 96, pp. 3429-3444.

Hui, C. Y., Jagota, A., Lin, Y. Y., Kramer, E. J. (2002) Constraints on microcontact printing imposed by stamp deformation. Langmuir, 18, pp. 1394-1407.

Liu, B., Yu, M. F., Huang, Y. (2004) Role of lattice registry in the full collapse and twist formation of carbon nanotubes. Phys. Rev. B, 70, pp. 161402.

Liu, J. L. (2009) Analogies between a meniscus and a cantilever. Chin. Phys. Lett., 26, pp. 116803.

Liu J L, Feng X Q. (2007) Hierarchical capillary adhesion of micro-cantilevers or hairs. J. Phys. D: Appl. Phys., 40, pp. 5564-5570.

Liu, J. L., Feng, X. Q. (2007) Capillary adhesion of microbeams: finite deformation analyses. Chin. Phys. Lett., 24, pp. 2349-2352.

Love, A.E.H. (1906) A treatise on the mathematical theory of elasticity, Second edition, Cambridge University Press, London.

Martel, R., Schmidt, T., Shea, H. R., Hertel, T., Avouris, P. (1998) Single- and multi-wall carbon nanotube field-effect transistors. Appl. Phys. Lett., 73, pp. 2447-2449.

Oyharcalbal, X., Frisch, T. (2005) Peeling off an elastica from a smooth attractive substrate. Phys. Rev. E, 71, pp. 036611.

Pantano, A., Parks, D. M., Boyce, M. C., Buongiorno Nardelli, M. Mixed finite element-tightbinding electromechanical analysis of carbon nanotubes. J. Appl. Phys., 96, pp. 67566760.

Poncharal, P., Wang, Z. L., Ugarte, D., de Heer, W. A. (1999) Electrostatic deflections and electromechanical resonances of Carbon Nanotubes. Science, 283, pp. 1513-1516.

Py, C., Reverdy, P., Doppler, L., Bico, J., Roman, B., Baroud, C. N. (2007) Capillary origami: spontaneous wrapping of a droplet with an elastic sheet. Phys. Rev. Lett., 98, pp. 156103.

Roman, B., Bico, J. (2010) Elasto-capillarity: deforming an elastic structure with a liquid droplet. J. Phys: Condens. Matter, 22, pp. 493101.

Ruoff, R. S., Tersoff, J., Lorents, D. C., Subramoney, S., Chan, B. (1993) Radial deformation of carbon nanotubes by van der Waals forces. Nature, 364, pp. 514-516.

Seifert, U., Lipowsky, R. (1990) Adhesion of vesicles. Phys. Rev. A, 42, pp. 4768-4771.

Tang, T., Glassmaker, N. J. (2010) On the inextensible elastica model for the collapse of nanotubes. Math. Mech. Solid, 15, pp. 591-606.

Tang, T., Jagota, A., Hui, C. (2005) Adhesion between single-walled carbon nanotubes. J. Appl. Phys., 97, pp. 074304.

Tang, T., Jagota, A., Hui, C. Y., Glassmaker, N. J. (2005) Collapse of single-walled carbon nanotubes. J. Appl. Phys., 97, pp. 074310.

Xiao, J., Liu, B., Huang, Y., Zuo, J., Hwang, K. C., Yu, M. F. (2007) Collapse and stability of single- and multi-wall carbon nanotubes. Nanotechnology, 18, pp. 395703.

Yu, M. F., Dyer, M. J., Chen, J., Qian, D., Liu, W. K., Ruoff, R. S. (2001) Locked twist in multiwalled carbon-nanotube ribbons. Phys. Rev. B, 64, pp. 241403.

$\mathrm{Yu}$, M. F., Kowalewski, T., Ruoff, R. S. (2000) Investigation of the radial deformability of individual carbon nanotubes under controlled indentation force. Phys. Rev. Lett., 85, pp. 1456-1459.

Zhao, Y. P., Wang, L. S., Yu, T. X. (2003) Mechanics of adhesion in MEMS: a review. J. Adhesion Sci. Technol., 17, pp. 519-546. 


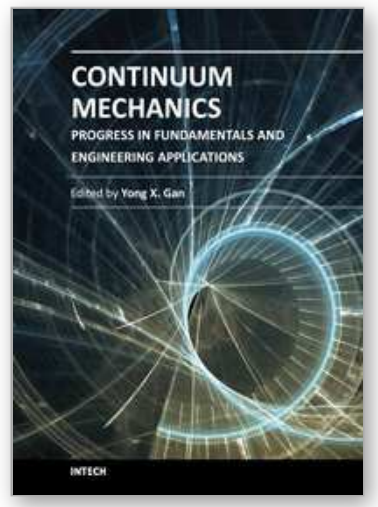

\author{
Continuum Mechanics - Progress in Fundamentals and \\ Engineering Applications \\ Edited by Dr. Yong Gan
}

ISBN 978-953-51-0447-6

Hard cover, 158 pages

Publisher InTech

Published online 28, March, 2012

Published in print edition March, 2012

Continuum Mechanics is the foundation for Applied Mechanics. There are numerous books on Continuum Mechanics with the main focus on the macroscale mechanical behavior of materials. Unlike classical Continuum Mechanics books, this book summarizes the advances of Continuum Mechanics in several defined areas. Emphasis is placed on the application aspect. The applications described in the book cover energy materials and systems (fuel cell materials and electrodes), materials removal, and mechanical response/deformation of structural components including plates, pipelines etc. Researchers from different fields should be benefited from reading the mechanics approached to real engineering problems.

\title{
How to reference
}

In order to correctly reference this scholarly work, feel free to copy and paste the following:

Jianlin Liu (2012). Transversality Condition in Continuum Mechanics, Continuum Mechanics - Progress in Fundamentals and Engineering Applications, Dr. Yong Gan (Ed.), ISBN: 978-953-51-0447-6, InTech, Available from: http://www.intechopen.com/books/continuum-mechanics-progress-in-fundamentals-and-engineeringapplications/transversality-condition-in-continuum-mechanics

\section{INTECH}

open science | open minds

\section{InTech Europe}

University Campus STeP Ri

Slavka Krautzeka 83/A

51000 Rijeka, Croatia

Phone: +385 (51) 770447

Fax: +385 (51) 686166

www.intechopen.com

\section{InTech China}

Unit 405, Office Block, Hotel Equatorial Shanghai

No.65, Yan An Road (West), Shanghai, 200040, China 中国上海市延安西路65号上海国际贵都大饭店办公楼 405 单元

Phone: +86-21-62489820

Fax: $+86-21-62489821$ 
(C) 2012 The Author(s). Licensee IntechOpen. This is an open access article distributed under the terms of the Creative Commons Attribution 3.0 License, which permits unrestricted use, distribution, and reproduction in any medium, provided the original work is properly cited. 